\title{
Analysis of an SEIS Epidemic Model with a Changing Delitescence
}

\author{
Jinghai Wang \\ Institute of Mathematics and Computer Science, Fuzhou University, Fuzhou, FuJian 350002, China \\ Correspondence should be addressed to Jinghai Wang, fzwjh@163.com
}

Received 17 May 2012; Accepted 26 July 2012

Academic Editor: Allan Peterson

Copyright (C) 2012 Jinghai Wang. This is an open access article distributed under the Creative Commons Attribution License, which permits unrestricted use, distribution, and reproduction in any medium, provided the original work is properly cited.

An SEIS epidemic model with a changing delitescence is studied. The disease-free equilibrium and the endemic equilibrium of the model are studied as well. It is shown that the disease-free equilibrium is globally stable under suitable conditions. Moreover, we also show that the unique endemic equilibrium of the system is globally asymptotically stable under certain conditions.

\section{Introduction}

Infectious diseases have tremendous influence on human life. Every year millions of human beings suffer from or die of various infectious diseases. It has been an increasingly complex issue to control infectious diseases. In order to predict the spreading of infectious diseases among regions, many epidemic models have been proposed and analyzed in recent years (see [1-14]). Bilinear and standard incidence rates have been frequently used in classical epidemiological models (see [5]). However, it is more effective by using nonlinear incidence. Mathematical models describing the population dynamics of infectious diseases have been playing an important role in disease control for a long time. Many scholars have studied mathematical models which describe the dynamical behavior of the transmission of infectious diseases (see also [1-14] and the references therein). A variety of nonlinear incidence rates have been used in epidemiological models (see [6-14]). However, the models with a changing delitescence have seldom been studied.

In recent years, the spread of infectious diseases is diversiform, such as H1N1 disease. The diversity of the delitescence period in each infected body who is infected with H1N1 virus is mainly due to the variation of the virus and the distinct constitution of different people. The study of the models with a changing delitescence plays an important role in controlling infectious diseases. In this paper, we consider an SEIS epidemic model with 
a changing delitescence and a nonlinear incidence rate, and we study the existence and stability of the equilibriums of the SEIS epidemic model.

By a standard nonlinear incidence rate $\beta S I /(1+\alpha I)$ and a changing delitescence $\mu$, we consider an SEIS epidemic model which consists of the susceptible individuals $(S)$, exposed individuals but not yet infected $(E)$, infectious individuals $(I)$, and the total population $(N)$.

The model is given as follows:

$$
\begin{aligned}
& S^{\prime}=A-d S-\frac{\beta S I}{1+\alpha I}+\gamma I, \\
& E^{\prime}=\mu \frac{\beta S I}{1+\alpha I}-(d+\varepsilon) E, \\
& I^{\prime}=(1-\mu) \frac{\beta S I}{1+\alpha I}+\varepsilon E-(d+\gamma+\delta) I,
\end{aligned}
$$

where $A$ is the recruitment rate of individuals (including newborns and immigrants) into the susceptible population; $d$ is the natural death rate; $\gamma$ is the rate at which infected individuals are treated or recovered; $\delta$ is disease-related death rate; $\varepsilon$ is the rate at which exposed individuals become infectious; $\mu$ is the rate at which infected individuals become exposed; $1-\mu$ is the rate at which infected individuals become infectious. The nonlinear incidence rate is assumed to be of the form $\beta S I /(1+\alpha I) . A, d, \gamma, \delta, \varepsilon$, and $\mu$ are all normal numbers with $0<\mu<1$. This, together with $N=S+E+I$, implies

$$
N^{\prime}=(S+E+I)^{\prime}=A-d N-\delta I .
$$

Thus, substituting $S=N-E-I$ and (1.2) into (1.1), we have

$$
\begin{aligned}
E^{\prime} & =\mu \frac{\beta I}{1+\alpha I}(N-E-I)-(d+\varepsilon) E \\
I^{\prime} & =(1-\mu) \frac{\beta S I}{1+\alpha I}(N-E-I)+\varepsilon E-(d+\gamma+\delta) I, \\
N^{\prime} & =A-d N-\delta I .
\end{aligned}
$$

Form (1.2), in the absence of the disease, that is, $I=0, N \rightarrow A / d$. Since the spread of the disease in the population will lead to the decrease of $N$, it follows that $N \in[0, A / d]$. Note that $D$ is a positively invariant region for the original model:

$$
D=\left\{(E, I, N) \mid E \geq 0, I \geq 0, N \geq 0, E+I \leq N \leq \frac{A}{d}\right\}
$$

and model (1.3) is obviously well-pased in $D$. 


\section{Existence of Equilibria}

Now, we study equilibria of model (1.3), (note that $m=d+\varepsilon, n=d+\gamma+\delta$ ). Steady states of model (1.3) satisfy the following equations:

$$
\begin{gathered}
\mu \frac{\beta I}{1+\alpha I}(N-E-I)-(d+\varepsilon) E=0, \\
(1-\mu) \frac{\beta S I}{1+\alpha I}(N-E-I)+\varepsilon E-(d+\gamma+\delta) I=0, \\
A-d N-\delta I=0 .
\end{gathered}
$$

If $I=0$, then $E=0$ and $N=A / d$, so (2.1) has the disease-free equilibrium $P_{0}(0,0, A / d)$.

If $I \neq 0$, from the third equation of (2.1), we obtain

$$
N=\frac{A}{d}-\frac{\delta}{d} I
$$

From the first and second equations of (2.1), we obtain

$$
E=\frac{\mu n}{m(1-\mu)+\mu \varepsilon} I .
$$

By substituting (2.2) and (2.3) into the first equation of (2.1), we obtain the following equation for $I$ :

$$
\left[\beta\left(\frac{\delta}{d}+1+\frac{\mu n}{m(1-\mu)+\mu \varepsilon}\right)+\frac{m n \alpha}{m(1-\mu)+\mu \varepsilon}\right] I=\frac{m n}{m(1-\mu)+\mu \varepsilon}\left[\frac{\beta A(m(1-\mu)+\mu \varepsilon)}{m n d}-1\right] .
$$

Let $R_{0}=\beta A(m(1-\mu)+\mu \epsilon) / m n d$.

It is easy to see that (2.4) has a positive root if and only if $R_{0}>1$. So (2.1) has a unique endemic equilibrium $P^{*}\left(E^{*}, I^{*}, N^{*}\right)$ with

$$
\begin{aligned}
I^{*} & =\frac{(m n /(m(1-\mu)+\mu \varepsilon))((\beta A(m(1-\mu)+\mu \varepsilon) / m n d)-1)}{\beta((\delta / d)+1+\mu n /(m(1-\mu)+\mu \varepsilon))+m n \alpha /(m(1-\mu)+\mu \varepsilon)}, \\
E^{*} & =\frac{\mu n}{m(1-\mu)+\mu \varepsilon} I^{*}, \quad N^{*}=\frac{A}{d}-\frac{\delta}{d} I^{*} .
\end{aligned}
$$

Then, we have the following theorem.

Theorem 2.1. If $R_{0} \leq 1$, model (1.3) only has the disease-free equilibrium $P_{0}(0,0, A / d)$; if $R_{0}>$ 1 , model (1.3) has a unique endemic equilibrium $P^{*}\left(E^{*}, I^{*}, N^{*}\right)$ except the disease-free equilibrium $P_{0}(0,0, A / d)$. 


\section{Stability of Equilibria}

Theorem 3.1. If $R_{0}<1$, then the disease-free equilibrium $P_{0}(0,0, A / d)$ is locally asymptotically stable; if $R_{0}=1, P_{0}(0,0, A / d)$ is stable; if $R_{0}>1, P_{0}(0,0, A / d)$ is unstable.

Proof. The linearization of model (1.3) about the equilibrium $P_{0}(0,0, A / d)$ gives

$$
\left|\begin{array}{ccc}
\lambda+m & -\frac{\mu \beta A}{d} & 0 \\
\varepsilon & \lambda-\frac{(1-\mu) \beta A}{d}+n & 0 \\
0 & \delta & \lambda+d
\end{array}\right|=0 .
$$

Thus, we have

$$
\left|\lambda E-J\left(P_{0}\right)\right|=(\lambda+d)\left[\lambda^{2}+\left(m+n-\frac{(1-\mu) \beta A}{d}\right) \lambda+m n-\frac{(1-\mu) m \beta A}{d}-\frac{\varepsilon \mu \beta A}{d}\right]=0 .
$$

Assume that $\lambda_{1}, \lambda_{2}$, and $\lambda_{3}$ are the roots of the above equation. Then, we know that $\lambda_{1}=-d<$ 0 , and $\lambda_{2}, \lambda_{3}$ are the roots of the following equation:

$$
\lambda^{2}+\left(m+n-\frac{(1-\mu) \beta A}{d}\right) \lambda+m n-\frac{(1-\mu) m \beta A}{d}-\frac{\varepsilon \mu \beta A}{d}=0
$$

According to Viete theorem, we have

$$
\lambda_{1}+\lambda_{2}=\frac{(1-\mu) m \beta A}{d}-m-n, \quad \lambda_{1} \lambda_{2}=m n\left(1-R_{0}\right) .
$$

If $R_{0}<1$, we have

$$
\lambda_{1}+\lambda_{2}=\frac{(1-\mu) m \beta A}{d}-m-n<\frac{m n(1-\mu)}{m(1-\mu)+\mu \varepsilon}-m-n<0, \quad \lambda_{1} \lambda_{2}=m n\left(1-R_{0}\right)>0 .
$$

It is easy to see that all the roots of (3.3) have negative real parts if and only if $R_{0}<1$. If $R_{0}=1$, it is obvious that one of eigenvalue of (3.3) is 0 . If $R_{0}>1$, one of the roots of (3.3) has positive real part. This completes the proof.

Theorem 3.2. If $R_{0}<1$, then the disease-free equilibrium $P_{0}(0,0, A / d)$ is globally asymptotically stable. 
Proof. Consider a Liapunov function as $V=\varepsilon E+m I$, then we have

$$
V^{\prime}=(m(1-\mu)+\mu \varepsilon) \frac{\beta I(N-E-I)}{1+\alpha I}-m n I \leq(m(1-\mu)+\mu \varepsilon) \beta \frac{A}{d} I-m n I=m n I\left(R_{0}-1\right) .
$$

If $R_{0}<1, V^{\prime} \leq 0$, then $V^{\prime}=0$ if and only if $E=I=0$.

Hence, according to Theorem 3.1, if $R_{0}<1$, the disease-free equilibrium $P_{0}(0,0, A / d)$ is globally asymptotically stable. This completes the proof.

Now, we study the local stability of the endemic equilibrium $P^{*}\left(E^{*}, I^{*}, N^{*}\right)$.

Substituting $E^{*}=(\mu n /(m(1-\mu)+\mu \varepsilon)) I^{*}$ into the first equation of $(2.1)$, we have

$$
\frac{\beta}{1+\alpha I^{*}}\left(N^{*}-E^{*}-I^{*}\right)=\frac{m n}{m(1-\mu)+\mu \varepsilon}
$$

Then, the Jacobi matrix of $(1.3)$ about $P^{*}\left(E^{*}, I^{*}, N^{*}\right)$ is

$$
J\left(P^{*}\right)=\left|\begin{array}{ccc}
-\frac{\mu \beta I^{*}}{1+\alpha I^{*}}-m & \frac{\mu}{1+\alpha I^{*}}\left[\frac{m n}{m(1-\mu)+\mu \varepsilon}-\beta I^{*}\right] & \frac{\mu \beta I^{*}}{1+\alpha I^{*}} \\
-\frac{(1-\mu) \beta I^{*}}{1+\alpha I^{*}}+\varepsilon & \frac{1-\mu}{1+\alpha I^{*}}\left[\frac{m n}{m(1-\mu)+\mu \varepsilon}-\beta I^{*}\right]-n & \frac{(1-\mu) \beta I^{*}}{1+\alpha I^{*}} \\
0 & -\delta & -d
\end{array}\right|
$$

Denote

$$
\begin{gathered}
j_{11}=-\frac{\mu \beta I^{*}}{1+\alpha I^{*}}-m, \quad j_{12}=\frac{\mu}{1+\alpha I^{*}}\left[\frac{m n}{m(1-\mu)+\mu \varepsilon}-\beta I^{*}\right], \quad j_{13}=\frac{\mu \beta I^{*}}{1+\alpha I^{*}}, \\
j_{21}=-\frac{(1-\mu) \beta I^{*}}{1+\alpha I^{*}}+\varepsilon, \quad j_{22}=\frac{1-\mu}{1+\alpha I^{*}}\left[\frac{m n}{m(1-\mu)+\mu \varepsilon}-\beta I^{*}\right]-n, \quad j_{23}=\frac{(1-\mu) \beta I^{*}}{1+\alpha I^{*}}, \\
j_{31}=0, \quad j_{32}=-\delta, \quad j_{33}=-d .
\end{gathered}
$$

According to [15], if $(1-\mu) \beta>\varepsilon \alpha$ and $m n /(m(1-\mu)+\mu \varepsilon) \leq \beta I^{*}$, then $m n / \beta(m(1-$ $\mu)+\mu \varepsilon) \leq I^{*} \leq \varepsilon /((1-\mu) \beta-\varepsilon \alpha)$. Note that $j_{i i}<0(i=1,2,3), j_{12} j_{21} \leq 0, j_{13} j_{31}=0, j_{23} j_{32}<0$, and then $J\left(P^{*}\right)$ is stable. 
If $(1-\mu) \beta>\varepsilon \alpha$ and $m n /(m(1-\mu)+\mu \varepsilon)>\beta I^{*}$, then $\varepsilon /((1-\mu) \beta-\varepsilon \alpha) \leq I^{*} \leq m n / \beta(m(1-$ $\mu)+\mu \varepsilon)$. Meanwhile, $\left((1-\mu) /\left(1+\alpha I^{*}\right)\right)\left[m n /(m(1-\mu)+\mu \varepsilon)-\beta I^{*}\right]-n<0$, that is,

$$
(n \alpha+(1-\mu) \beta) I^{*}>0>\frac{(1-\mu) m n}{m(1-\mu)+\mu \varepsilon}-n=-\frac{n \mu \varepsilon}{m(1-\mu)+\mu \varepsilon},
$$

hence, $j_{i i}<0(i=1,2,3), j_{12} j_{21} \leq 0, j_{13} j_{31}=0, j_{23} j_{32}<0$, which proves that $J\left(P^{*}\right)$ is stable.

If $(1-\mu) \beta \leq \varepsilon \alpha$ and $m n /(m(1-\mu)+\mu \varepsilon)>\beta I^{*}$, to obtain $j_{i i}<0 \quad(i=1,2,3), j_{12} j_{21} \leq 0$, $j_{13} j_{31}=0, j_{23} j_{32}<0$, there must be $\varepsilon \leq((1-\mu) \beta-\varepsilon \alpha) I^{*}$, but it is impossible.

From the above discussion, we get the following conclusion.

Theorem 3.3. If $R_{0}>1$, system (1.3) has a unique endemic equilibrium $P^{*}\left(E^{*}, I^{*}, N^{*}\right)$, which is locally asymptotically stable.

From the third equation of (1.3), we have $N^{\prime}=A-d N-\delta I \leq A-d N$. Note that $I \rightarrow 0$ as $t \rightarrow \infty$, then $N(t) \rightarrow A / d$ as $t \rightarrow \infty$. The limit system of (1.3) is

$$
\begin{aligned}
& E^{\prime}=\mu \frac{\beta I}{1+\alpha I}\left(\frac{A}{d}-E-I\right)-m E \\
& I^{\prime}=(1-\mu) \frac{\beta I}{1+\alpha I}\left(\frac{A}{d}-E-I\right)+\varepsilon E-n I .
\end{aligned}
$$

It is easy to see that system (3.11) has a disease-free equilibrium $\bar{P}_{0}(0,0)$. If $R_{0}>1$, system (3.11) has a unique endemic equilibrium $\bar{P}^{*}\left(\bar{E}^{*}, \bar{I}^{*}\right)$ with

$$
\begin{aligned}
I^{*} & =\frac{(m n /(m(1-\mu)+\mu \varepsilon))(\beta A(m(1-\mu)+\mu \varepsilon) / m n d-1)}{\beta(1+\mu n /(m(1-\mu)+\mu \varepsilon))+m n \alpha /(m(1-\mu)+\mu \varepsilon)}, \\
E^{*} & =\frac{\mu n}{m(1-\mu)+\mu \varepsilon} I^{*} .
\end{aligned}
$$

Consider the Dulac function $B=1 / I$. Note that

$$
\begin{aligned}
& P=\mu \frac{\beta I}{1+\alpha I}\left(\frac{A}{d}-E-I\right)-m E, \\
& Q=(1-\mu) \frac{\beta I}{1+\alpha I}\left(\frac{A}{d}-E-I\right)+\varepsilon E-n I,
\end{aligned}
$$

then $\partial(B P) / \partial(E)+\partial(B Q) / \partial(I)=-\mu(\beta /(1+\alpha I))-m / I-\left((1-\mu) \beta /(1+\alpha I)^{2}\right)(-(1+\alpha I)-$ $\alpha(A / d-E-I))-\left(\varepsilon / I^{2}\right) E<0$, there is no limit cycle in the first quadrant of the $I-E$ plane. System (3.11) has a unique endemic equilibrium $\bar{P}^{*}\left(\bar{E}^{*}, \bar{I}^{*}\right)$, then we prove that $\bar{P}^{*}\left(\bar{E}^{*}, \bar{I}^{*}\right)$ is globally asymptotically stable.

From the above discussion, we get the following conclusion.

Theorem 3.4. There is no limit cycle, and the endemic equilibrium $P^{*}\left(E^{*}, I^{*}, N^{*}\right)$ of system (3.11) is globally asymptotically stable. 


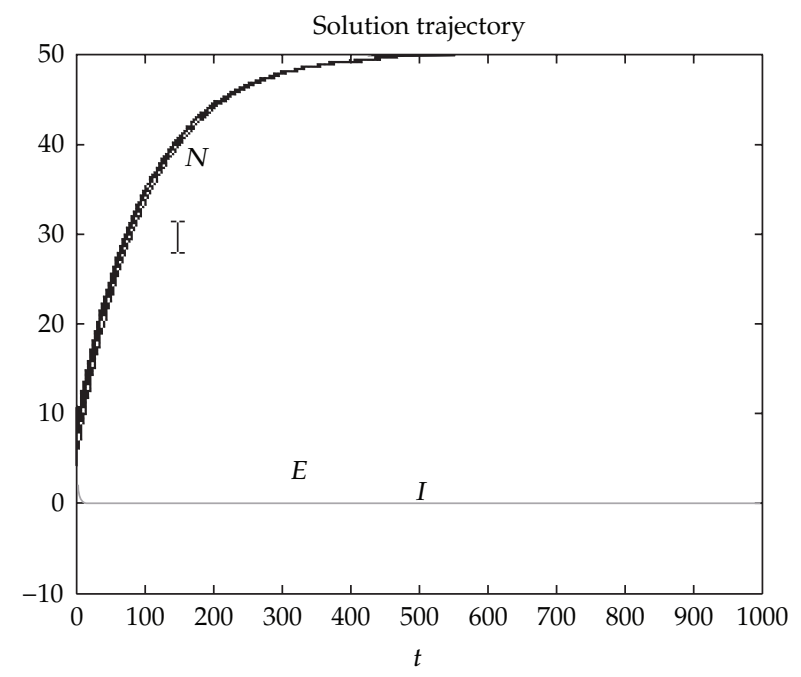

Figure 1: Dynamical behavior of system (1.3) with $\mu=0.9$.

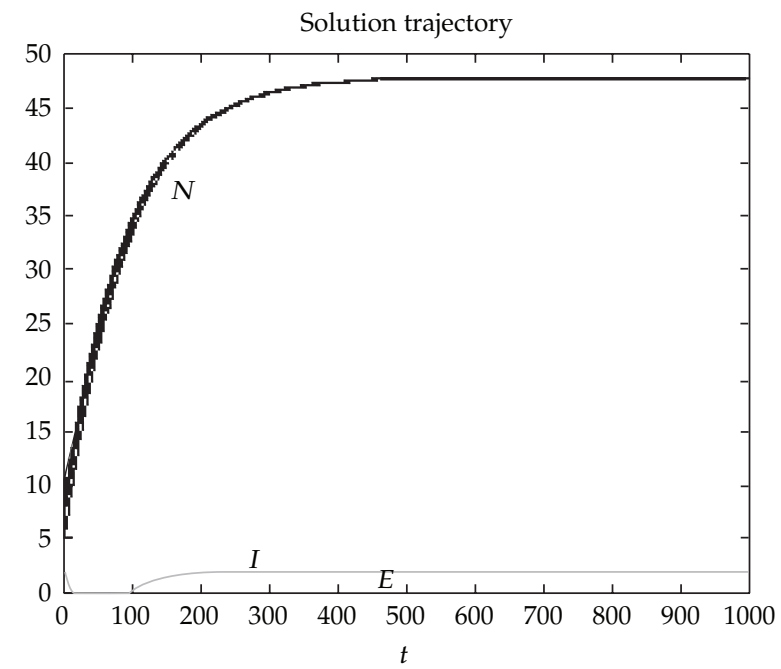

Figure 2: Dynamical behavior of system (1.3) with $\mu=0.001$.

When $t \rightarrow \infty, P^{*}\left(E^{*}, I^{*}, N^{*}\right) \rightarrow P^{*}\left(E^{*}, I^{*}, A / d\right)$. It is easy to see that the stability of $P^{*}\left(E^{*}, I^{*}, A / d\right)$ is equivalent to that of $\bar{P}^{*}\left(\bar{E}^{*}, \bar{I}^{*}\right)$. Since $\bar{P}^{*}\left(\bar{E}^{*}, \bar{I}^{*}\right)$ is globally asymptotically stable, the unique endemic equilibrium $P^{*}\left(E^{*}, I^{*}, N^{*}\right)$ is also globally asymptotically stable.

\section{Numerical Simulation}

Figures 1-5 are drawn by MATLAB. With different parameters, they describe the changes of the number of exposed individuals who are not yet infectious, infectious individuals, and the total population. 


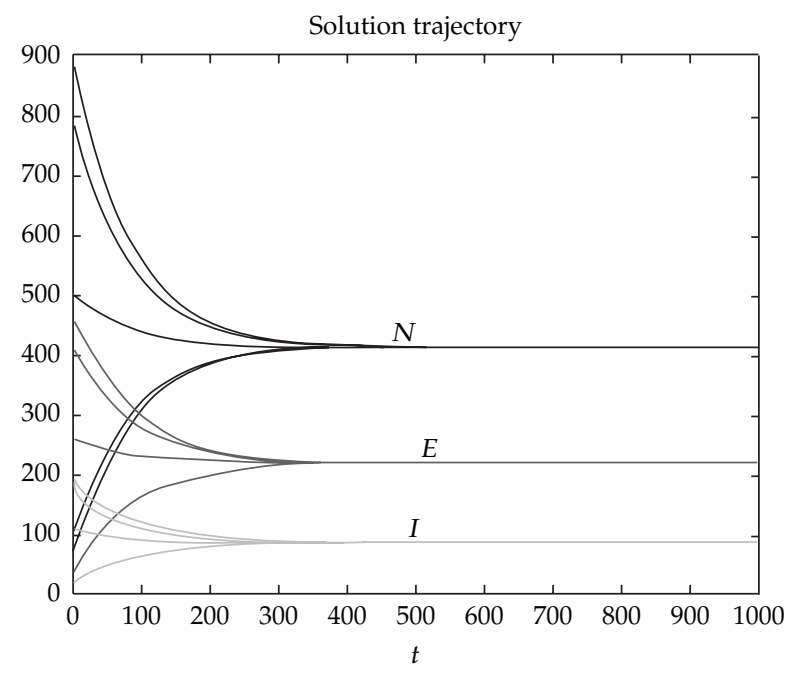

Figure 3: Dynamical behavior of system (1.3) with $\mu=0.99$.

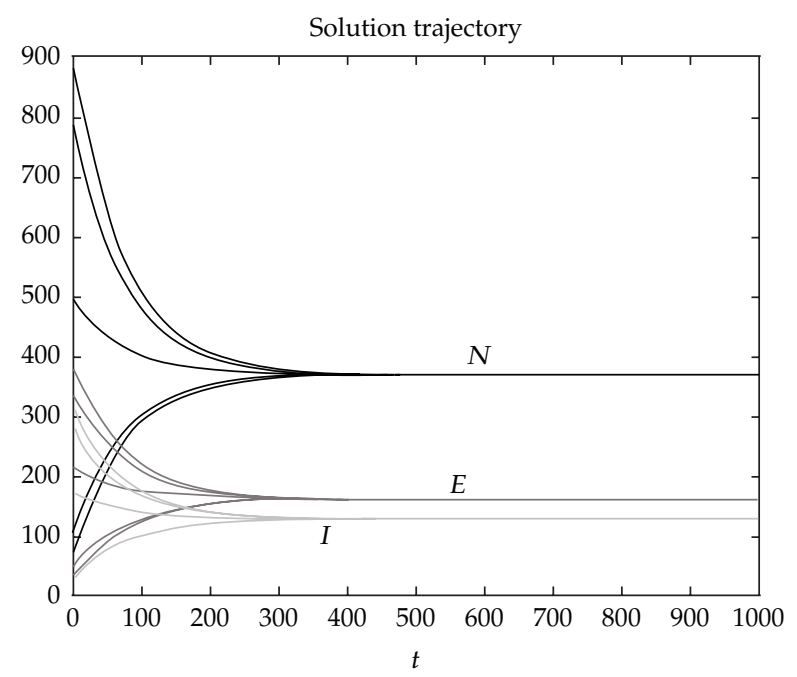

Figure 4: Dynamical behavior of system (1.3) with $\mu=0.5$.

With $\beta=0.02, d=0.01, A=0.5, m=0.4, n=0.5, \varepsilon=0.1, \alpha=0.4, \delta=0.01$, we consider $(2,3,5),(3,2,7),(2,2,5),(3,4,8),(2,5,9),(5,4,9),(2,4,9)$ as the initial values. Figure 1 describes system (1.3) with $\mu=0.9\left(R_{0}=0.65\right)$, Figure 2 describes system (1.3) with $\mu=0.001$ $\left(R_{0}=1.99\right)$.

For the initial values $(20,30,500),(30,20,700),(20,20,500),(30,40,800),(20,50,900)$, $(50,40,900),(20,40,900)$, Figures 3,4 , and 5 are drawn with $\beta=0.5, d=0.01, A=5, m=0.3$, $n=0.5, \varepsilon=0.1, \alpha=0.4, \delta=0.01$ by MATLAB. With $\mu=0.99\left(R_{0}=170\right), \mu=0.5\left(R_{0}=333.33\right)$, and $\mu=0.01\left(R_{0}=496.67\right)$, Figures $3-5$ describe the endemic equilibriums of system (1.3).

From Figures 1 and 2, it is easy to see that the value of $\mu$ decides the development trend of the infectious disease, that is, whether the infectious disease dies out or does not exist forever when the other conditions are the same. 


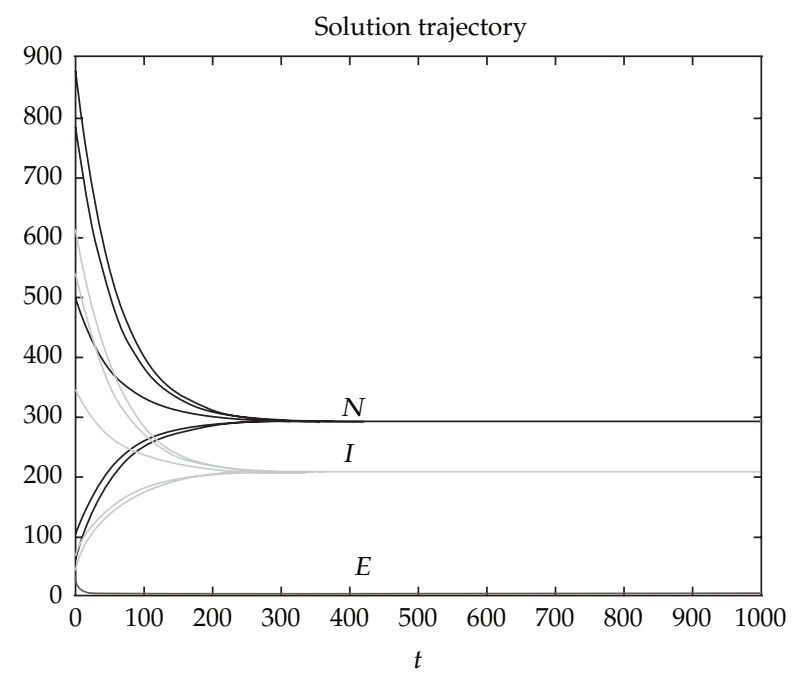

Figure 5: Dynamical behavior of system (1.3) with $\mu=0.01$.

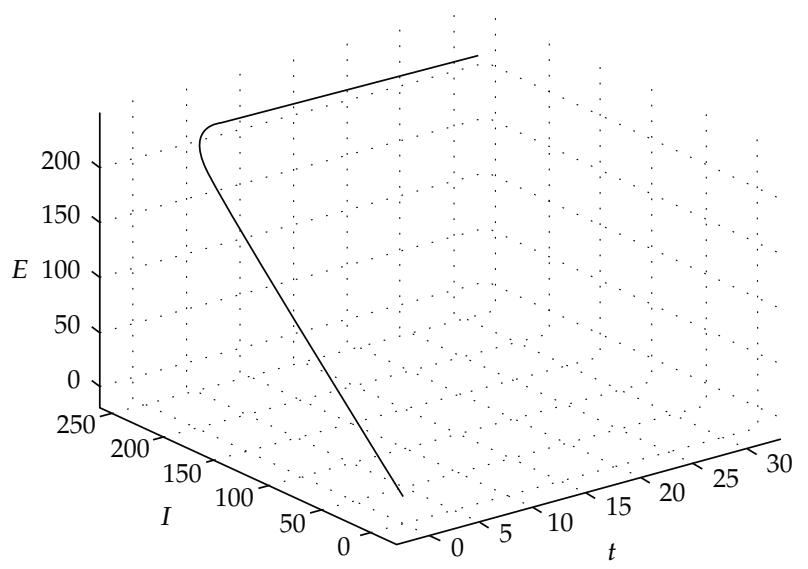

Figure 6: Dynamical behavior of system (3.11).

From Figures 3-5, with a unique endemic equilibrium of system (1.3), the value of $\mu$ will affect the numbers of exposed individuals who are not yet infectious, infectious individuals, and the total population.

With $\beta=0.5, d=0.01, m=0.3, n=0.5, \mu=0.5, \alpha=0.4, \varepsilon=0.1$, Figure 6 shows that system (3.11) has no limit cycle.

\section{Conclusion}

Because of distinct constitutions of individuals, some infected individuals who are not yet infectious become exposed individuals, while other infected individuals immediately become infectious. So, we establish an epidemic model with a changing delitescence between SEIS and SIS model by using the proportional number $\mu$. 
This paper mainly considers the existence and stability of equilibriums. We use Jacobi matrix to discuss the local asymptotical stability of the endemic equilibrium, and we obtain sufficient conditions for this. When we discuss its global asymptotical stability, we use the Dulac function in its limit system (3.11), and we get relatively complete conclusions.

After the discussion of the stability of the equilibriums, we have made numerical simulations of the epidemic model with different values of $\mu$. By discussing the basic reproduction number $R_{0}=\beta A(m(1-\mu)+\mu \varepsilon) / m n d$, we show that the value of $\mu$ is important in the tendency of the infectious diseases with the other conditions being the same. We must pay attention to the changing delitescence, develop effective control strategies, then we can better control the tendency of infectious diseases.

\section{References}

[1] E. Beretta, T. Hara, W. Ma, and Y. Takeuchi, "Global asymptotic stability of an SIR epidemic model with distributed time delay," Nonlinear Analysis: Theory, Methods E Applications, vol. 47, no. 6, pp. 4107-4115, 2001.

[2] Y. Takeuchi, W. Ma, and E. Beretta, "Global asymptotic properties of a delay SIR epidemic model with finite incubation times," Nonlinear Analysis: Theory, Methods E Applications, vol. 42, no. 6, pp. 931-947, 2000.

[3] R. M. Anderson and R. M. May, "Population biology of infectious diseases: part 1," Nature, vol. 280, pp. 361-367, 1979.

[4] L. Q. Gao, J. Mena-Lorca, and H. W. Hethcote, "Four SEI endemic models with periodicity and separatrices," Mathematical Biosciences, vol. 128, no. 1-2, pp. 157-184, 1995.

[5] Z. Ma, Y. Zhou, W. Wang, and Z. Jin, Mathematical Ecology, Springer, New York, NY, USA, 1990.

[6] S. Ruan and W. Wang, "Dynamical behavior of an epidemic model with a nonlinear incidence rate," Journal of Differential Equations, vol. 188, no. 1, pp. 135-163, 2003.

[7] D. Xiao and S. Ruan, "Global analysis of an epidemic model with nonmonotone incidence rate," Mathematical Biosciences, vol. 208, no. 2, pp. 419-429, 2007.

[8] W. M. Liu, H. W. Hethcote, and S. A. Levin, "Dynamical behavior of epidemiological models with nonlinear incidence rates," Journal of Mathematical Biology, vol. 25, no. 4, pp. 359-380, 1987.

[9] A. d'Onofrio, "Stability properties of pulse vaccination strategy in SEIR epidemic model," Mathematical Biosciences, vol. 179, no. 1, pp. 57-72, 2002.

[10] S. Liao, "On the homotopy analysis method for nonlinear problems," Applied Mathematics and Computation, vol. 147, no. 2, pp. 499-513, 2004.

[11] Y. R. Shi, X. J. Xu, Z. X. Wu et al., "Application of the homotopy analysis method to solving nonlinear evolution equations," Acta Physica Sinica, vol. 55, no. 4, pp. 1555-1560, 2006.

[12] J. Hui and D. Zhu, "Global stability and periodicity on SIS epidemic models with backward bifurcation," Computers \& Mathematics with Applications, vol. 50, no. 8-9, pp. 1271-1290, 2005.

[13] J. Li and Z. Ma, "Qualitative analyses of SIS epidemic model with vaccination and varying total population size," Mathematical and Computer Modelling, vol. 35, no. 11-12, pp. 1235-1243, 2002.

[14] L.-I. Wu and Z. Feng, "Homoclinic bifurcation in an SIQR model for childhood diseases," Journal of Differential Equations, vol. 168, no. 1, pp. 150-167, 2000.

[15] C. Jeffries, V. Klee, and P. van den Driessche, "When is a matrix sign stable?" Canadian Journal of Mathematics, vol. 29, no. 2, pp. 315-326, 1977. 


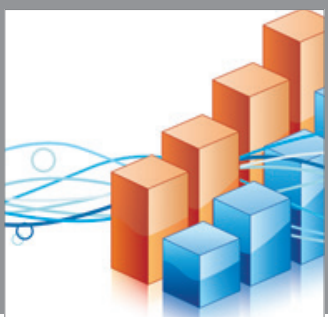

Advances in

Operations Research

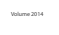

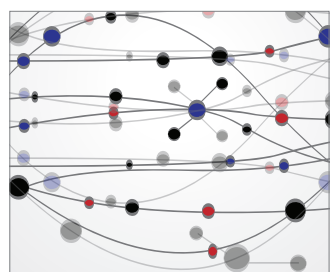

\section{The Scientific} World Journal
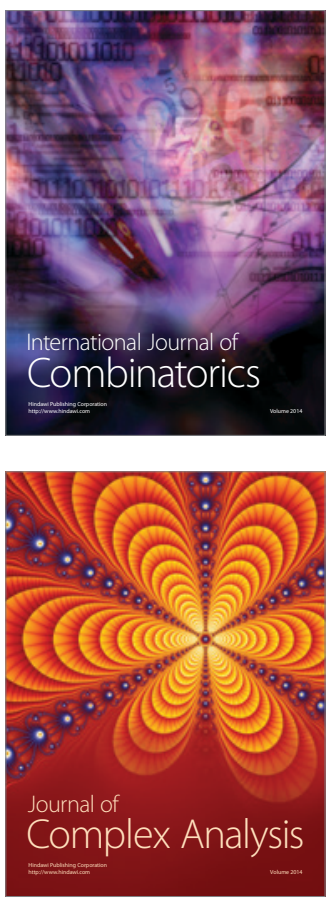

International Journal of

Mathematics and

Mathematical

Sciences
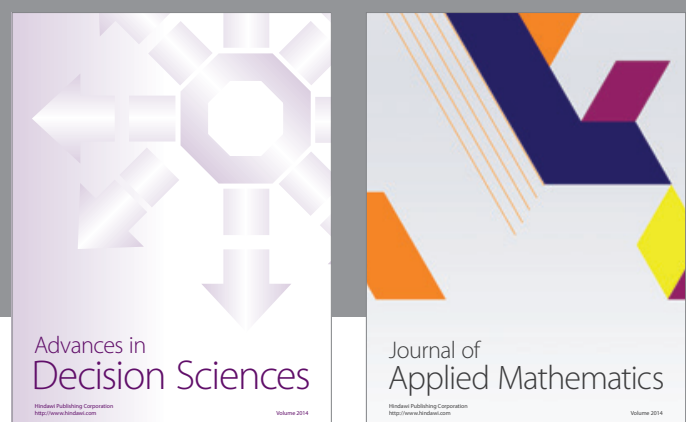

Journal of

Applied Mathematics
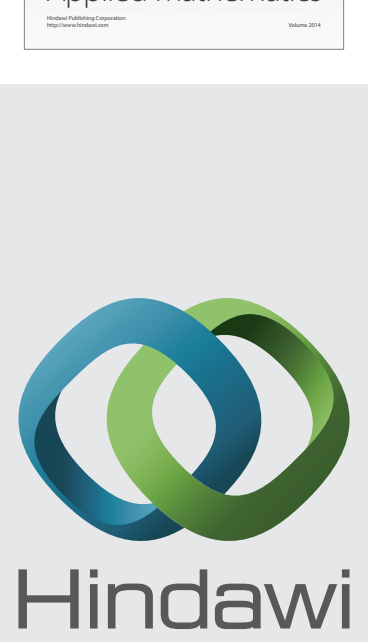

Submit your manuscripts at http://www.hindawi.com
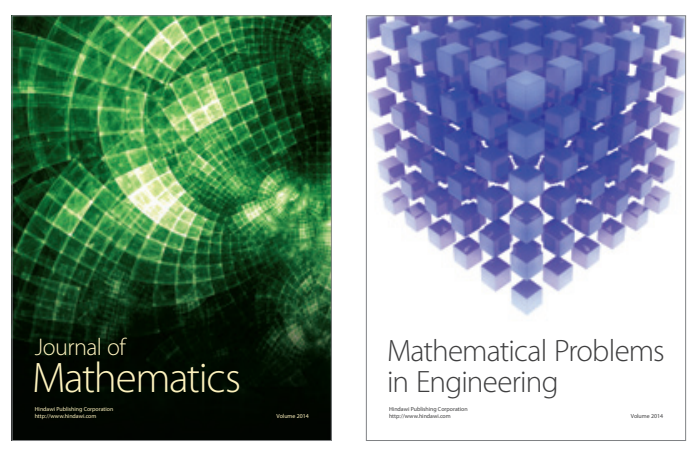

Mathematical Problems in Engineering
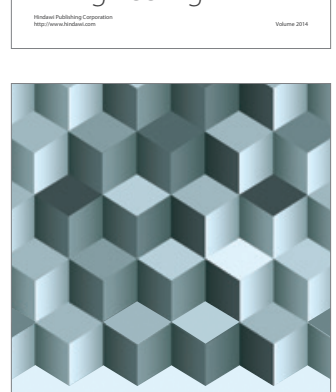

Journal of

Function Spaces
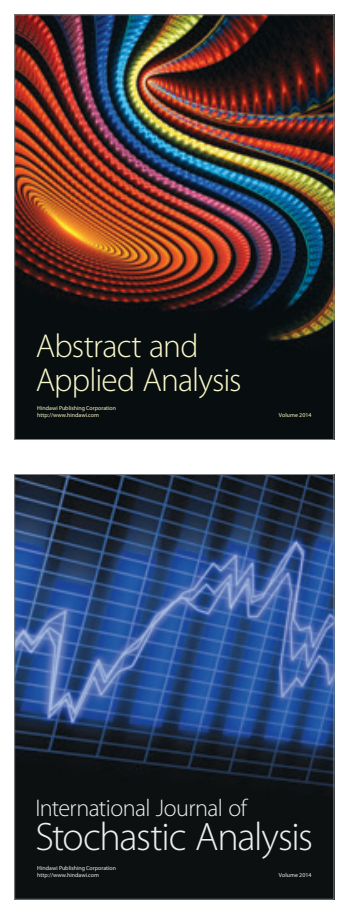

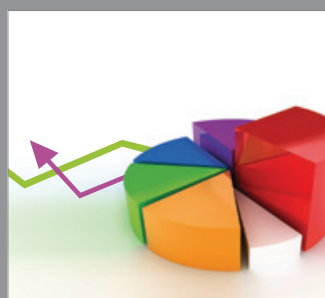

ournal of

Probability and Statistics

Promensencen
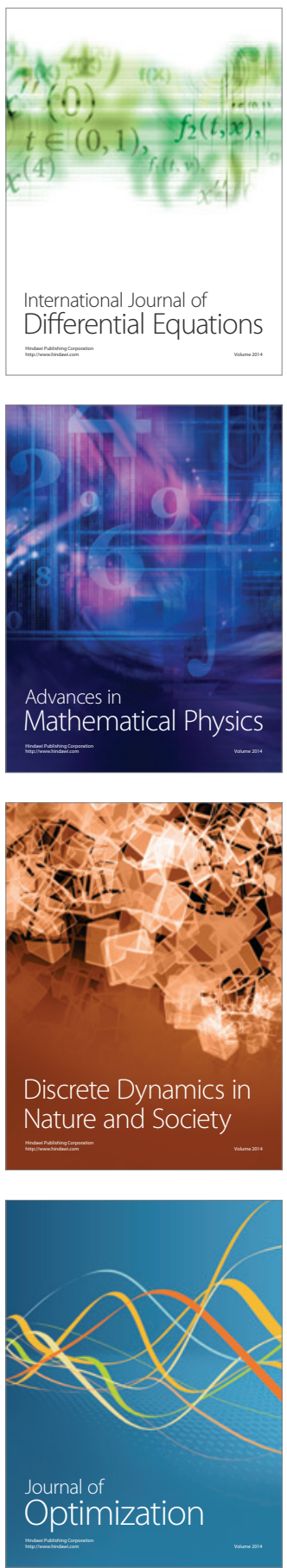